\title{
HPV-58 Molecular Variants Exhibit Different Transcriptional Activity
}

\author{
Tainá Raiol ${ }^{a}$ Regina Maria Santos de Amorim ${ }^{a}$ Pedro Galante ${ }^{b}$ \\ Cláudia Renata Fernandes Martins ${ }^{a, \dagger}$ Luisa Lina Villa ${ }^{b}$ Laura Sichero ${ }^{b}$ \\ ${ }^{a}$ Department of Cellular Biology, Institute of Biology, University of Brasilia, ICC Sul, Brasília, and \\ ${ }^{b}$ Department of Virology, Ludwig Institute for Cancer Research, São Paulo, Brazil
}

\section{Key Words}

Human papillomavirus • Molecular variants, HPV •

Transcription $\cdot$ Sequencing

\begin{abstract}
Early promoter activity of HPV-58 molecular variants isolated from high-grade cervical lesions in Brazil was compared. Luciferase reporter assays were conducted in C33 cells transfected with the complete long control region of 3 molecular variants of HPV-58 as well as HPV-58, -18 or -16 prototypes. The HPV-58 prototype and Bsb-329 and Bsb-367 variants showed a promoter activity similar to that of HPV-16, but lower than that of Bsb-295 and HPV-18. The introduction of the Bsb-295 7788 mutation into the HPV-58 prototype resulted in the enhancement of transcription closer to Bsb-295 and HPV-18. These results could impact the expression of E6 and E7 viral oncogenes.

Copyright $\odot 2010$ S. Karger AG, Basel
\end{abstract}

Human papillomavirus (HPV) infection is a cause of cervical cancer [1]. HPV-16, the most prevalent type worldwide, is detected in about $40 \%$ of high-grade cervical lesions [2]. The incidence of other high-risk HPV types differs among countries and even throughout re- gions of the same country [3]. HPV-58 is the second most frequent type of cervical lesions, not only in the Federal District of Central Brazil [4], but also in other countries of Latin America and Asia [5-7]. Differences in homology of $<2 \%$ within the L1 gene characterize HPV molecular variants [8]. Nevertheless, nucleotide variability among variants can be as high as $5 \%$ in the long control region (LCR) [9]. The central segment of the LCR encloses an epithelial cell-specific enhancer important for the regulation of viral replication and transcription of early genes. Cellular factors like AP-1, GRE, NF-1, Oct1, Sp1, TEF-1, and YY1 have been shown to impact on HPV promoter activity [10].

HPVs 16 and 18 intratype variability studies revealed important features concerning viral evolution, infection persistence and progression to clinically relevant lesions [11]. Molecular variants of both HPV types cluster into phylogenetic groups that reflect the evolution and migration patterns of humans [9]. Conversely, phylogenetic trees of HPVs 31, 35, 52 and 58 did not evidence human

GenBank accession No.: HPV-18 prototype ID:X05015; HPV-16 prototype ID:K02718; HPV-58 prototype ID:D90400; Bsb-295 ID:FJ202010; Bsb-329 ID:FJ202011, and Bsb-367:FJ202012.

\section{KARGER}

Fax +41613061234 E-Mail karger@karger.ch www.karger.com
(C) 2010 S. Karger AG, Basel

0300-5526/11/0543-0146\$38.00/0

Accessible online at:

www.karger.com/int
Laura Sichero

Department of Virology, Ludwig Institute for Cancer Research

São Paulo Branch, Rua João Julião, 245

01323-903, São Paulo, SP (Brazil)

Tel. +55 113388 3212, Fax +55 113284 5311, E-Mail lsichero@ ludwig.org.br 
evolution and spread to the extent described for HPVs 16 and 18 [12]. In addition, data regarding the epidemiological and functional significance of molecular variants of less frequent high-risk HPV types are still limited. We have recently described nucleotide variability within the LCR, and E6 and L1 genes of HPVs 31, 33, 35, 52 and 58 in high-grade cervical lesions in the Federal District [13]. This analysis indicated HPV-58 as the most conserved in all genome regions analyzed. The complete LCR sequence of $8 \mathrm{HPV}-58$ samples characterized 3 molecular variants. Since nucleotide alterations within the LCR could influence the location and binding affinity of cellular and viral transcriptional factors, we aimed to compare E6/E7 promoter activity of the variants detected.

The complete LCR region (nucleotide positions 7140 115) from the HPV-58 prototype and 3 variants were amplified, and cloned within the pGL3-Basic vector upstream of the luciferase gene (Promega, Madison, Wisc., USA). Sequencing results were confirmed in at least 2 independent polymerase chain reactions in order to exclude errors introduced during DNA amplification. The HPV-58mut sequence was generated using the GeneTailor ${ }^{\mathrm{TM}}$ Site-Directed Mutagenesis System (Invitrogen, Carlsbad, Calif., USA).

The human cervical HPV-negative cancer cells C33 (ATCC HTB-31) were maintained in Dulbecco's modified Eagle's medium supplemented with $10 \%$ fetal calf serum and antibiotics. Cells were co-transfected using lipofectamine (Invitrogen) in 10-cm diameter dishes with 4 $\mu \mathrm{g}$ of recombinant plasmid LCR-pGL3, and $1 \mu \mathrm{g}$ of the pCMV- $\beta-$ Gal plasmid used as an internal control of transfection efficiency. For comparison, plasmids enclosing HPVs 18 or 16 prototype LCR sequences were also analyzed [14]. Each LCR activity was tested in 7 independent experiments in triplicate. Cells were harvested $48 \mathrm{~h}$ after transfection with $900 \mu l$ Reporter Lysis Buffer (Promega). The Promega Luciferase Assay System and the Promega $\beta$-Galactosidase Enzyme Assay were used to evaluate luciferase and $\beta$-galactosidase activities in proteins extracts, respectively.

Measurements of relative luciferase activity were normalized for those of $\beta$-galactosidase activity and protein amount obtained for the same protein extract. Average values were based on the mean of triplicates of 7 independent experiments. Wilcoxon rank-sum test was used to determine if relative luciferase activity was significantly different among individual variants and HPV types. $p$ values of $<0.05$ were considered significant. Statistical analyses were carried out using program $\mathrm{R}$ (http://www. r-project.org).

Transcriptional Activity of HPV-58 Variants
Figure 1 depicts nucleotide variability within the LCR of three natural HPV-58 variants. The HPV-58 prototype was cloned from a squamous cervical carcinoma [15] that has only been detected in Japan [12]. High conservation of the HPV-58 LCR sequence is reflected by the detection of only 4 substitutions within a 794-bp fragment. The mutation at position 7714 detected in all HPV-58 variants overlaps putative binding sites for the transcription factors NF-1 and C/EBP, as indicated using the TRANSFAC database [16]. In addition, nucleotide position 7788, mutated only in the Bsb-295 variant, overlaps a potential binding site for the viral E2 protein. Although the outcome of these mutations upon the activity of the binding sites is still unknown, they may impact the expression of E6/E7 oncoproteins.

The functional significance of HPV-58 LCR variability was estimated in C33 cells. Luciferase measurements were normalized to $\beta$-galactosidase activity, and we observed that none of the HPV constructs alone affected $\beta$-galactosidase function. Transcriptional activity of the HPV-16 prototype was arbitrarily defined as the reference. As expected, early promoter of HPV-18 was more active than that of HPV-16 [14]. Figure 2 summarizes promoter activity measurements of HPV-58 prototype and molecular variants. Overall, there was significant variability across molecular variants with respect to their transcriptional activity. HPV-58 prototype and variants Bsb-329 and Bsb-367 showed promoter activity similar to HPV-16 but about sixfold lower than HPV-18. Interestingly, HPV-58 variant Bsb-295 presented high promoter activity, even comparable to HPV-18. Since a substitution in nucleotide position 7788 was only observed in the Bsb295 variant, we sought to make a point mutant in the background of the HPV-58 prototype sequence (HPV58 mut). Extensive sequencing of the full-length LCR was performed to certify that this was the only nucleotide change created. We observed that the introduction of this single nucleotide alteration led to a 3.2-fold enhancement of the transcriptional activity as compared to HPV-58 prototype. Differences among HPV-58mut, Bsb-295 and HPV-18 were not statistically significant, although all 3 were different from the other constructs tested. Nevertheless, HPV-58mut presented lower transcriptional activity in all 7 independent experiments as compared to Bsb-295. Taken together, these data indicate that the enhanced transcriptional activity observed for the Bsb-295 isolate could be ascribed, at least to some extent, to nucleotide position 7788 .

The International Agency for Research on Cancer classifies $13 \mathrm{HPV}$ types as carcinogenic in humans due to 


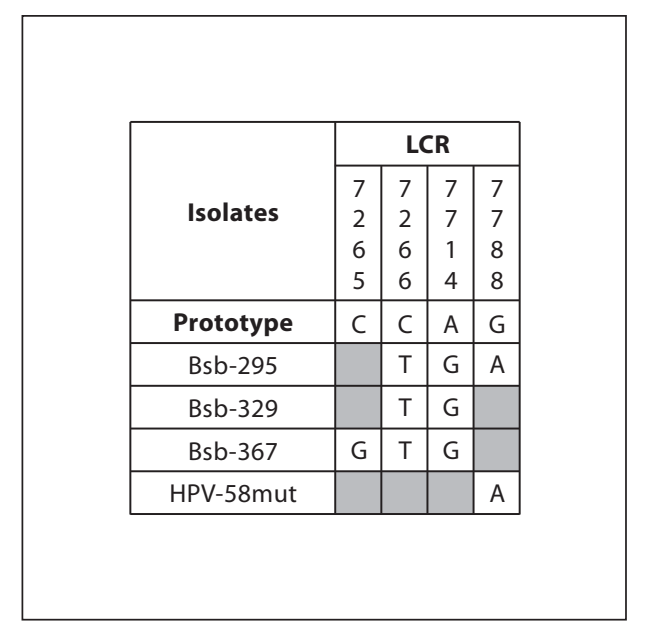

Fig. 1. Genetic variability within the long control region (LCR) of HPV-58 molecular variants. Nucleotide positions where variations were detected are written vertically across the top. In grey, positions at which no variations were identified relative to HPV58 prototype, whereas a letter indicates a variant nucleotide at this position. In the HPV-58mut isolate, a substitution in nucleotide position 7788 was generated by site-directed mutagenesis in the background of the HPV-58 prototype sequence.

their association with cervical cancer development [3]. Owing to the high prevalence of HPVs 16 and 18 worldwide, genome variability has been widely studied for both viral types. It has been described that, in admixtured populations of the American continents, non-European variants of HPVs 16 and 18 are more prone to persist and are also associated with an increased risk of cervical lesion development [17-19]. Furthermore, some studies suggested that functionally non-European variants of HPVs 16 and 18 are more oncogenic as compared to European isolates as a result of higher promoter activity [14, 20], higher efficiency in replication [21], and biological differences among E6/E7 proteins [22-24].

Worldwide HPV-58 was detected in $2 \%$ of cervical cancers [25]. HPV-58 together with HPVs 16, 31, 33, 35, 52 and 67 form HPV species 9 of the Alpha-papillomavirus genus [8]. Interestingly, in the present study, both HPVs 16 and 58 prototypes presented similar promoter activities.

Sequence analysis of a LCR fragment from $101 \mathrm{HPV}$ 58-positive samples collected worldwide identified 21 molecular variants, none corresponding to the prototype genome [12] which is highly prevalent in Asia [5, 26]. Based on HPV-58 E7 variability, it was observed that specific variants showed a positive trend of association with

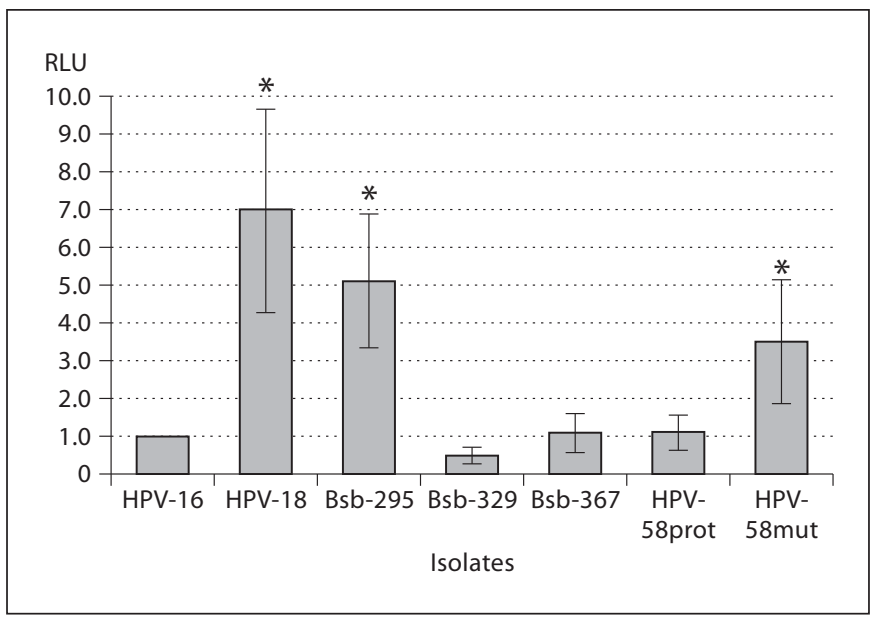

Fig. 2. Relative luciferase units (RLU) detected among HPV-58 variants and HPVs 58, 16 or 18 prototypes. Transcriptional activity was normalized to that of the HPV-16 prototype which was arbitrarily defined as the reference and set to a value of 1.0. The mean values of 7 independent assays \pm SD are illustrated. The Wilcoxon test was used to make pair-wise comparison between isolates. Asterisks indicate constructs which show similar transcriptional activity statistically different from all others $(p<0.05$, Wilcoxon test).

the severity of neoplasia among samples from Hong Kong [27]. Since the E7 sequence of the samples examined in the present study was not analyzed, any comparison to the data of Chan et al. [27] is precluded. Nevertheless, we also sequenced the E6 gene of these isolates and detected a single substitution at nucleotide position 307 that was common to all variants analyzed. However, this alteration was not associated with clinical outcome in samples from Korean women [28].

In the present study, we analyzed the effect on promoter activity of sequence variations in the LCR of HPV58 isolates from high-grade cervical lesion patients living in Central Brazil. Nucleotide variability within this sequence was comparatively low relative to that of other high-risk HPV types $[12,13]$. Despite the small number of nucleotide changes within the LCR, differences were observed in the transcriptional activity among molecular variants. The Bsb-295 variant, which presented the highest transcriptional activity among all variants tested, is identical to the Bsb-329 variant with the exception of the substitution at nucleotide position 7788 . Nevertheless, the Bsb-329 variant presented a significantly lower activity. The introduction of this mutation in the background of the HPV-58 prototype sequence resulted in the enhancement of promoter activity. Although the 7788 posi- 
tion overlaps a presumed binding site for the E2 protein, transfections were conducted in a HPV-negative cell line, so we discard the influence of this viral protein in the results obtained. Nevertheless, the effect of this substitution upon binding of other transcription factors to this region or adjacent recognition sequences is still unknown. Additional studies to further verify the impact of this nucleotide alteration in E6/E7 expression and to identify cellular factors involved in this regulation are warranted.

This is the first report describing transcriptional activities of different HPV-58 molecular variants. The Bsb295 variant presented the highest promoter activity among all isolates tested. The single 7788 nucleotide alteration observed in this variant was capable of enhancing viral promoter activity and could lead to higher expression of E6 and E7 viral genes in vivo. Nevertheless, in order to attribute a higher oncogenic potential to this variant, several studies should investigate whether this variant is more prone to persist, more associated with disease development, and also has a better transforming potential in cell culture. The Bsb-295 variant was detected in 2 of 8 samples analyzed in Central Brazil. This variant could also be particularly prevalent in other geographic regions where HPV-58 has frequently been associated with cervical cancer development.

\section{Acknowledgements}

Plasmids containing HPV-16 or HPV-18 prototype sequence genomes were kindly provided by E.M. de Villiers. Plasmid containing HPV-58 prototype sequence genome was kindly provided by T. Matsukura. T.R. received a fellowship from CNPq. We are grateful to Dr. Marcelo de Macedo Brígido for all technical assistance at the University of Brasilia.

\section{References}

1 Walboomers JM, Jacobs MV, Manos MM, Bosch FX, Kummer JA, Shah KV, Snijders PJ, Peto J, Meijer CJ, Munoz NJ: Human papillomavirus is a necessary cause of invasive cervical cancer worldwide. J Patho 1999;189: 12-19.

-2 Clifford GM, Smith JS, Aguado T, Franceschi S: Comparison of HPV type distribution in high-grade cervical lesions and cervical cancer: a meta-analysis. Br J Cancer 2003;89: 101-105.

3 IARC Monographs on the Evaluation of Carcinogenic Risks to Humans. Vol 90: Human Papillomaviruses. Lyon, IARC, 2007.

-4 Camara GNL, Cerqueira DM, Oliveira APG, Silva EO, Carvalho LGS, Martins CRF: Prevalence of human papillomavirus types in women with pre-neoplastic and neoplastic cervical lesions in the Federal District of Brazil. Mem Inst Oswaldo Cruz 2003;98:879883.

5 Chan PKS, Li WH, Ma WI, Cheung JLK, Cheng AF: High prevalence of human papillomavirus type 58 in Chinese women with cervical cancer and pre-cancerous lesions. J Med Virol 1999;59:232-238.

-6 Giuliano AR, Papenfuss M, Abrahamsen M, Denmanc C, de Zapien JG, Henzen JLN, Ortega L, de Gala EMB, Stephan J, Feng J, Baldwin S, Garcia F, Hatch K: Human papillomavirus infection at the United StatesMexico border: implications for cervical cancer prevention and control. Cancer Epidemiol Biomarkers Prev 2001;10:1129-1136.
Sasagawa T, Basha W, Yanazaki H, Inoue M: High-risk and multiple human papillomavirus infections associated with cervical abnormalities in Japanese women. Cancer Epidemiol Biomarkers Prev 2001;10:45-52.

8 de Villiers EM, Fauquet C, Broker TR, Bernard HU, zur Hausen H: Classification of papillomaviruses. Virology 2004;324:17-27.

9 Ho L, Chan SY, Burk RD, Das BC, Fuginaga K, Icenogle JP, Kahn T, Kiviat N, Lancaster W, Mavromara-Nazos P, Labropoulou V, Mitrani-Rosenbaum S, Norrild B, Pillai MR, Stoerker J, Syrjaenen K, Syrjaenen S, Tay SK, Villa LL, Wheeler CM, Williamson AL, Bernard HU: The genetic drift of human papillomavirus type 16 is a means of reconstructing prehistoric viral spread and the movement of ancient human populations. J Virol 1993;67:6413-6423.

10 Bernard HU: Gene expression of genital human papillomaviruses and considerations on potential antiviral approaches. Antivir Ther 2002;7:219-237.

11 Sichero L, Villa LL: Epidemiological and functional implications of molecular variants of human papillomavirus. Braz J Med Biol Res 2006;39:707-717.

12 Calleja-Macias IE, Villa LL, Prado JC, Kalantari M, Allan B, Williamson AL, Chung, LP, Collins RJ, Zuna RE, Dunn ST, Chu TY, Cubie HA, Cuschieri K, von Knebel-Doeberitz M, Martins CR, Sanchez GI, Bosch FX, Munoz N, Bernard HU: Worldwide genomic diversity of the high-risk human papillomavirus types $31,35,52$, and 58 , four close relatives of human papillomavirus type 16. J Virol 2005;79:13630-13640.
13 Raiol T, Wyant PS, Amorim RMS, Cerqueira DM, Milanezi NG, Brígido MM, Sichero L, Martins CRF: Genetic variability and phylogeny of the high-risk HPV-31, -33, -35, -52 and -58 in Central Brazil. J Med Virol 2009; 81:685-692.

14 Sichero L, Franco EL, Villa LL: Different P105 promoter activities among natural variants of human papillomavirus type 18. J Infect Dis 2005; 191:739-742.

15 Matsukura T, Sugase M: Molecular cloning of a novel human papillomarvirus (type 58) from an invasive cervical carcinoma. Virology 1990;177:833-836.

16 Wingender E, Dietze P, Karas H, Knuppel R: TRANSFAC: a database on transcription factors and their DNA binding sites. Nucleic Acids Res 1996;24:238-241.

17 Berumen J, Ordoñez RM, Lazcano E, Salmeron J, Galvan SC, Estrada RA, Yunes E, Garcia-Carranca A, Gonzales-Lira G, De La Campa AM: Asian-American variants of human papillomavirus type 16 and risk for cervical cancer: a case-control study. J Natl Cancer Inst 2001;93:1325-1330.

- 18 Hildesheim A, Schiffman M, Bromley C, Wacholder S, Herrero R, Rodriguez A, Bratti MC, Sherman ME, Scarpidis U, Lin QQ, Terai M, Bromley RL, Buetow K, Apple RJ, Burk RD: Human papillomavirus type 16 variants and risk of cervical cancer. J Natl Cancer Inst 2001;93:315-318.

19 Sichero L, Ferreira S, Trottier H, DuarteFranco E, Ferenczy A, Franco EL, Villa LL: High grade cervical lesions are caused preferentially by non-European variants of HPVs 16 and 18. Int J Cancer 2007;120:1763-1768. 
>20 Kämmer C, Warthorst U, Torrez-Martinez $\mathrm{N}$, Wheeler CM, Pfister H: Sequence analysis of the long control region of human papillomavirus type 16 variants and functional consequences for P97 promoter activity. J Gen Virol 2000;81:1975-1981.

-21 Hubert WG: Variant upstream regulatory region sequences differentially regulate human papillomavirus type 16 DNA replication throughout the viral life cycle. J Virol 2005;79:5914-5922.

-22 Contreras-Paredes A, De la Cruz-Hernandez E, Martinez-Ramirez I, Duenas-Gonzalez A, Lizano M: E6 variants of human papillomavirus 18 differentially modulate the protein kinase B/phosphatidylinositol 3-kinase (akt/ PI3K) signaling pathway. Virology 2009;383: 78-85.
23 Stöppler MC, Ching K, Stöppler H, Clancy K, Schlegel R, Icenogle J: Natural variants of human papillomavirus type 16 E6 protein differ in their abilities to alter keratinocyte differentiation and to induce p53 degradation. J Virol 1996;70:6987-6993.

24 Zehbe I, Richard C, DeCarlo CA, Shai A, Lambert PF, Lichtig H, Tommasino M, Sherman L: Human papillomavirus 16 E6 variants differ in their dysregulation of human keratinocyte differentiation and apoptosis. Virology 2009;383:69-77.

25 Bosch FX, Muñoz N, de Sanjosé S, Guerrero E, Gaffari AM, Kaldor J, Castellsagué X, Shah KV: Importance of human papillomavirus endemicity in incidence of cervical cancer: an extension of hypothesis on sexua behavior. Cancer Epidemiol Biomarkers Prev 1999;3:375-379.
26 Xin CY, Matsumoto K, Yoshikawa H, Yasugi T, Onda T, Nakagawa S, Yamada M, Nozawa S, Sekiya S, Hirai Y, Shiromizu K, Fujii T, Taketani Y: Analysis of E6 variants of human papillomavirus type 33, 52 and 58 in Japanese women with cervical intraepithelial neoplasia/cervical cancer in relation to their oncogenic potential. Cancer Lett 2001;170: 19-24.

27 Chan PKS, Lam CW, Cheung TH, Li WH, Lo KWK, Chan MYM, Cheng AF, Cheung JLK: Association of human papillomavirus type 58 variant with the risk of cervical cancer. J Natl Cancer Inst 2002;94:1249-1253.

28 Bae JH, Cheung JL, Lee SJ, Luk AC, Tong SY, Chan PK, Park JS: Distribution of human papillomavirus type 58 variants in progression of cervical dysplasia in Korean women. J Microbiol Biotechnol 2009;19:1051-1054. 